\title{
Atypical clinical presentation of COVID-19: a case of Guillain-Barrè Syndrome related to SARS-Cov-2 infection
}

\author{
Angelo Benvenuto, Angelo Michele Carella*, Matteo Conte, Francesco Damone, Teresa Marinelli, Cinzia \\ Florio, Pasquale De Luca, Michele Di Pumpo, Giovanni Modola, Giuseppe Ciavarrella and Martino Nargiso \\ Department of Internal Medicine, "T Masselli Mascia” Hospital, 71016 San Severo (Foggia), Italy
}

*Corresponding author: Angelo Michele Carella, Department of Internal Medicine,

“T Masselli Mascia” Hospital, Italy.

Received Date: May 01, 2020

Published Date: May 14, 2020

\begin{abstract}
In these months the diffusion of a novel beta coronavirus, known as Severe Acute Respiratory Syndrome Coronavirus 2 (SARS-CoV-2), is causing a worldwide public health emergency originated in Wuhan, China. SARS-CoV-2 infection may develop asymptomatic or begin with mild flu-like symptoms, but in severe case it causes the so called "Coronavirus Disease 2019" (COVID-19), characterized by serious interstitial pneumonia that may quickly develop into severe acute respiratory distress syndrome (ARDS), septic shock, sepsis-induced coagulopathy and fatal multi organ dysfunction.
\end{abstract}

Emerging evidence indicates that SARS-CoV-2 infection can also cause neurological manifestations. In this report we describe an atypical clinical presentation of COVID-19, started as Guillain-Barré syndrome (GBS) and without typical respiratory symptoms of SARS-Cov-2 disease.

Keywords: COVID-19; Guillain-Barrè Syndrome; SARS-CoV-2 infection

\section{Introduction}

In these months the diffusion of a novel beta coronavirus, known as Severe Acute Respiratory Syndrome Coronavirus 2 (SARS-CoV-2), is causing a worldwide public health emergency originated in Wuhan, China. The novel coronavirus was reported to cause symptoms resembling the severe acute respiratory syndrome (SARS-CoV) by previous coronavirus in the years 2002 and 2003. Genetic sequencing of the virus suggests that it is closely linked to the SARS coronavirus. Both share the same receptor, angiotensinconverting enzyme 2 (ACE2) and therefore this virus was named SARS-CoV-2 [1, 2].

SARS-CoV-2 is very contagious and its rapid propagation has spread globally; there are three main transmission routes of COVID-19 infection: droplets, contact and aerosol transmission [3]. The gold standard for diagnosis of SARS-CoV-2 infection is real-time polymerase chain reaction fluorescence (RT-PCR) for detecting SARS-CoV-2 nucleic acid in samples of sputum or throat swab and in secretions of upper respiratory tract. Other potential diagnostic method might be the detection of specific IgM and IgG antibodies against SARS-Cov-2 in blood samples, although this method seems more appropriate for population screening [4].

The most prevailing onset symptoms of this infection, after an approximate incubation period of five days on average, are fever, cough, myalgia and fatigue, but also diarrhea, leg pain, dysgeusia and hyposmia $[5,6]$. Although most patients infected by SARSCoV-2 are asymptomatic or develop mild to moderate symptoms, a subset of patients develops serious interstitial pneumonia that may quickly progress to severe acute respiratory distress syndrome (ARDS), septic shock and fatal multi organ dysfunction that are the most severe clinical manifestations of SARS-Cov-2 infection [7].

High serum levels of Interleukin-6 (IL-6) and D-Dimer seem closely related to the occurrence of severe COVID-19 and their combined detection may be very useful for early prediction of severe COVID-19 patients; moreover, the patients present frequently lymphopenia and neutrophilia, hypoalbuminemia, high serum levels of alanine aminotransferase, lactate dehydrogenase, C-reactive protein, and sudden oxygenation deterioration [8]. 
Given that acute respiratory syndrome is the hallmark feature of severe COVID-19, most initial studies have focused on its impact on the respiratory system. However, accumulating evidence suggests that SARS-CoV-2 also infects other organs and can affect various body systems [9]. The expression and distribution of ACE2 in multiple human organs, including nervous system and skeletal muscles, suggests that SARS-CoV-2 might have a neuroinvasive potential and its impact on the nervous system might occur through direct infection or via secondary effects relating to intense systemic inflammatory response linked to viral infection [10-12]. Indeed, in severe cases of COVID-19 it has been shown a massive release of proinflammatory mediators and cytokines, in particular Interleukin-6 (IL-6) and Interleukin-1 (IL-1), linked to viral replication and leading to cytokine release syndrome-like [13].

Recent retrospective data from China showed that $36 \%$ of 214 SARS-CoV-2 infected patients had neurological manifestations, including acute cerebrovascular disease and impaired consciousness [14]; in addition, a first case of encephalitis with SARS-CoV-2 RNA detection in cerebrospinal fluid (CSF) was reported [15].

In this case report we describe an atypical clinical presentation of COVID-19, started as Guillain-Barré Syndrome (GBS) and without typical respiratory symptoms of SARS-Cov-2 disease.

\section{Case Report}

A 62-years- old male patient was admitted in our Internal Medicine Unit, complaining for some days of acute progressive symmetric weakness started in distal lower extremities and progressed to proximal limbs. Neurological manifestations were associated with pain, paraesthesias, peripheral oedema, severe fatigue and serious functional limitation in the movements. The patient denied fever, cough, respiratory symptoms or diarrhea and his past medical history was unremarkable. Previous corticosteroid treatment was already started few days before.

At admission, the patient had not fever nor dyspnea and was conscious; blood pressure was $120 / 75 \mathrm{mmHg}$, heart rate 110 beats/ minute and oxygen saturation $98 \%$ on air; clinical examination was normal except for asymmetric weakness in all limbs, presenting $1 / 5$ value of Medical Research Council scale at lower extremities and $2 / 5$ value at upper extremities, without cranial nerves involvement.

No abnormalities were found in chest-X-ray, transthoracic echocardiogram and abdominal ultrasonography; electrocardiogram showed sinus tachycardia (105 beats/minute). The patient underwent cervical and brain magnetic resonance imaging that revealed normal finding except for enhancement of the nerve roots.

Abnormal laboratory tests were found as following: high serum levels of C-reactive protein (447 mg/l), erythrocyte sedimentation rate $(92 \mathrm{~mm} /$ hour$)$, ferritin $(1857 \mathrm{ng} / \mathrm{ml})$, procalcitonin $(8,7 \mathrm{ng} /$ $\mathrm{ml}$ ), lactate dehydrogenase (574 IU/l), D-dimer (935 ng/ml), glucose (211 mg/dl), fibrinogen (1013 mg/dl), myoglobin (702 ng/ $\mathrm{ml}$ ) and Troponin I-hs (72 ng/l); severe hypoalbuminemia (1.57 $\mathrm{g} / \mathrm{dl}$ ), mild normocytic nomochromic anemia, thrombocytopenia
$(69000 / \mu \mathrm{l})$ and marked lymphocytopenia $(260 / \mu \mathrm{l})$ with normal white cells count $(9200 / \mu \mathrm{l})$ were also observed. No abnormalities were found in peripheral smear except poor platelets, aPTT and PT/ INR values were in normal range and blood gas analysis revealed respiratory alkalosis with high lactates (3.3 mmol/l) and normal oxygen saturation.

Non-organ specific auto-antibodies (ANA, AMA, ENA, dsDNA, ANCA) resulted negative as well as anti-HIV test and antiviral antibodies against Epstein-Barr virus, Cytomegalovirus, Herpesvirus, Togavirus, and hepatitis C and B markers; both urine and blood cultures were negative. CFS analysis by lumbar puncture revealed normal cells count and lack of albumin-cytological dissociation.

Given that GBS was suspected, the patient started therapy based on intravenous immunoglobulin (IGIV $0.4 \mathrm{~g} / \mathrm{kg}$ for a planned 5-day course), steroid therapy (Methylpredisolone $1 \mathrm{mg} / \mathrm{kg}$ ) and subcutaneous Enoxaparin (6000 IU daily).

Considering the laboratory abnormalities and the COVID-19 outbreak we decided to search SARS-Cov- 2 by subjecting the patient to nasopharyngeal swab which resulted positive on RT-PCR assay. The patient was transferred to Infectious Deseases Unit where he continued IGIV therapy and began treatment with tocilizumab, hydroxychloroquine and plasmapheresis. The patient currently continues hospitalization in this clinical setting.

\section{Discussion}

In this study, we report a case of atypical infection of SARSCoV-2 initially occurred as acute GBS. GBS is immune-mediated demyelinating disease of the peripheral nerves and nerve roots (polyradiculoneuropathy) that is usually triggered by various infections. At the moment six pathogens have been associated with GBS in case-control studies: Campylobacter Jejuni, Cytomegalovirus, Hepatitis E virus, Mycoplasma Pneumoniae, Epstein-Barr virus and Zika virus. Although the clinical presentation of the disease is heterogeneous, the classic manifestations of GBS are progressive, ascending and symmetrical flaccid paralysis of limbs, along with areflexia or hyporeflexia and with or without cranial nerve involvement. Pain is frequently reported and can be muscular, radicular or neuropathic [16].

Disease onset is acute or subacute and can progress over days to a few weeks. Diagnosis of GBS is based on the patient history and neurological, electrophysiological and CSF examinations. The classic finding in GBS is the combination in the CSF of elevated protein levels and normal cell count, known as albumin-cytological dissociation. However, protein levels are normal in $30-50 \%$ of patients in the first week after disease onset and normal CSF protein levels do not rule out a diagnosis of GBS [16, 17].

Emerging evidence indicates that SARS-CoV-2 infection may cause neurological complications and some cases of GBS associated with SARS-CoV-2 infection have been recently observed in Italy, China and in other countries; in these cases the interval of 5 to 10 days observed between the onset of viral illness and the first 
symptoms of GBS resulted similar to the interval observed in GBS cases that occur during or after other infections. In one case, fever and respiratory symptoms developed 7 days after the onset of GBS symptoms so that parainfectious profile pattern of GBS, instead of classic post-infectious profile, was suggested [18-21]. In our case the patient never showed respiratory symptoms nor fever; laboratory abnormalities, in particular high inflammatory parameters, lymphocytopenia and thrombocytopenia, suggested an infectious disease such as SARS-CoV-2.

The link between viral infection and neurological manifestations is not yet clear; neurotropic and neuroinvasive capabilities of other coronaviruses such as SARS-CoV and MERS-CoV were described in humans and the neurological manifestations included encephalitis, polyneuropathy and GBS $[22,23]$. The SARS-Cov-2 impact on the nervous system could be through direct infection or via secondary effects relating to intense systemic inflammatory response linked to viral infection $[2,11]$. Recent report of GBS associated with SARSCoV-2 raises concern for this virus to be a possible trigger [19]. It may be hypothesized that an aberrant immune response to the infection determines a serious inflammatory damage to peripheral nerves with molecular mimicry reaction, although the pathogenesis in not fully understood $[16,24]$.

We speculate that SARS-CoV-2 infection may be responsible for GBS development in this patient; we think SARS-Cov-2 may stimulate inflammatory cells causing massive release of pro-inflammatory mediators and cytokines, triggering immune-mediated neuropathy. Among various hypotheses it cannot be excluded that SARS-CoV-2 may generate auto-antibodies against specific gangliosides.

\section{Conclusion}

Apart asymptomatic patients, awareness of atypical clinical presentation of SARS-Cov-2 infection is remarkable and essential to avoid its contagious spread, particularly on hospital admission. This clinical case suggests the need to also consider potential neurological manifestations of COVID-19 and physicians should consider the potential link between GBS and SARS-CoV-2 infection. Therefore, during this epidemic era of COVID-19, to ensure SARSCoV-2 infection is never overlooked, clinical symptoms of GBS should be considered in COVID-19 differential diagnosis to avoid delayed diagnosis or misdiagnosis.

\section{Acknowledgement}

None.

\section{Conflict of Interest}

The Authors declare no conflict of interest.

\section{References}

1. Zhun N, Zhang D, Wang W, Li X, Yang B, et al. (2020) A novel coronavirus from patients with pneumonia in China. N Engl J Med 382(8): 727-733.

2. Zhao Y, Zhao Z, Wang Y, Zhou Y, Ma Y, et al. (2020) Single-cell RNA expression profiling of ACE2, the putative receptor of Wuhan 2019-nCov.

3. National Health Commission of People's Republic of China (2020) Notice of the General Office of the National Health and Health Commission on issuing a new coronavirus pneumonia prevention and control plan $\left(4^{\text {th }}\right.$ edn).

4. Yuen KS, Ye ZW, Fung SY, Chan CP, Jin DY (2020) SARS-CoV-2 and COVID-19: The most important research questions. Cell Biosci 10: 40.

5. Huang C, Wang Y, Li X, Ren L, Ren L, Zhao J, et al. (2020) Clinical features of patients infected with 2019 novel coronavirus in Wuhan,China. Lancet 395(1023): 497-506.

6. Vaira LA, Salzano G, Deiana G, De Riu G (2020) Anosmia and ageusia: common findings in COVID-19 patients. Laryngoscope 10.1002/ lary.28692.

7. Yang X, Yu Y, Xu J, Shu H, Xia J, et al. (2020) Clinical course and outcomes of critically ill patients with SARS-CoV-2 pneumonia in Wuhan, China: a single-centered, retrospective, observational study. Lancet Respir Med $8(4):$ e26.

8. Gao Y, Li T, Han M, Li X, Wu D, et al. (2020) Diagnostic Utility of Clinical Laboratory Data Determinations for Patients with the Severe COVID-19. J Med Virol pp: 1-6.

9. Sahin AR, Erdogan A, Agaoglu PM, Dineri Y, Kakirci AY, et al. (2020) Novel Coronavirus COVID-Outbreak: A Review of the Current Literature. EJMO 4(1): 1-7.

10. Hamming I, Timens W, Bulthuis ML, Lely AT, Navis G, et al. (2004) Tissue distribution of ACE2 protein, the functional receptor for SARS coronavirus: a first step in understanding SARS pathogenesis. J Pathol 203(2): 631-637.

11. De Felice FG, Tovar Moll F, Moll J, Munoz DP, Ferreira ST (2020) Severe Acute Respiratory Syndrome Coronavirus 2(SARS-CoV-2) and the Central Nervous System. Trends Neurosci.

12. Pleasure SJ, Green AJ, Josephson SA (2020) The spectrum of neurologic disease in the Severe Acute Respiratory Syndrome Coronavirus 2 pandemic Infection: neurologists move to the frontlines. JAMA Neurol.

13. Zhang C, Wu Z, Li JW, Zhao H, Wang GQ (2020) The cytokine release syndrome (CRS) of severe COVID-19 and Interleukin-6 receptor (IL6R) antagonist Tocilizumab may be the key to reduce the mortality. Int J Antimicrob Agents 105954.

14. Mao L, Jin H, Wang M, Hu Y, Chen S, et al (2020) Neurological manifestations of hospidalized patients with coronavirus disease 2019 in Wuhan, China. JAMA Neurol pp. e201127.

15. Takeshi M, Norikazu H, Junko G, Harada D, Sugawara H, et al (2020) A first case of meningitis/encephalitis associated with SARS-Coronavirus-2. Int J Infect Dis 94: 55-58.

16. Leonhard SE, Mandarakas MR, Gondim FAA, Bateman K, Ferreira MLB, et al (2019) Diagnosis and management of Guillan-Barré syndrome in ten steps. Nat Rev Neurol 15(11): 671-683.

17. Sejvar JJ, Baughman AL, Wise M, Morgan OW (2011) Population incidence of Guillain-Barré syndrome: a systematic review and metaanalysis. Neuroepidemiology 36: 123-33.

18. Toscano G, Palmerini F, Ravaglia S, Ruiz L, Invernizzi P, et al. (2020) Guillain Barré syndrome associated with SARS-CoV-2. N Engl J Med pp. c2009191.

19. Virani A, Rabold E, Hanson T, Haag A, Elrufay R, et al. (2020) GuillainBarré syndrome associated with SARS-CoV-2 infection. IDCases e00771.

20. Sedaghat Z, Karimi N (2020) Guillain Barré syndrome associated with COVID-19 infection: a case report. J Clin Neurosci S09675868(20)30882-1.

21. Zhao H, Shen D, Zhou H, Liu J, Chen S (2020) Guillain-Barré syndrome associated with SARS-CoV-2 infection: causality or coincidence? Lancet Neurol 19(5): 383-384.

22. Bohmwald K, Galvez N, Rios M, Kalergis AM (2018) Neurological alterations due to respiratory virus infection. Front Cell Neurosci 12: 386. 
23. Kim JE, HeoJH, Kim Ho, Song SH, Park SS, et al. (2017) Neurological Complications during Treatment of Middle East Respiratory Syndrome. J Clin Neurol 13(3): 227-233.
24. Jacobs BC, Rothbarth PH, Van der Mechè FG, Herbrink P, Schmitz PI, et al (1998) The spectrum of antecedent infection in Guillain-Barré syndrome: a case control study. Neurology 51(4): 1110-1115. 